ARTICLE

Received 1 Dec 2014 | Accepted 18 Aug 2015 | Published 24 Sep 2015

DOI: $10.1038 /$ ncomms 9392

OPEN

\title{
Diffusional spread and confinement of newly exocytosed synaptic vesicle proteins
}

Niclas Gimber ${ }^{1}$, Georgi Tadeus ${ }^{1}$, Tanja Maritzen ${ }^{1}$, Jan Schmoranzer ${ }^{1,3}$ \& Volker Haucke ${ }^{1,2,3}$

Neurotransmission relies on the calcium-triggered exocytic fusion of non-peptide neurotransmitter-containing small synaptic vesicles (SVs) with the presynaptic membrane at active zones (AZs) followed by compensatory endocytic retrieval of SV membranes. Here, we study the diffusional fate of newly exocytosed SV proteins in hippocampal neurons by high-resolution time-lapse imaging. Newly exocytosed SV proteins rapidly disperse within the first seconds post fusion until confined within the presynaptic bouton. Rapid diffusional spread and confinement is followed by slow reclustering of SV proteins at the periactive endocytic zone. Confinement within the presynaptic bouton is mediated in part by SV protein association with the clathrin-based endocytic machinery to limit diffusional spread of newly exocytosed SV proteins. These data suggest that diffusion, and axonal escape of newly exocytosed vesicle proteins, are counteracted by the clathrin-based endocytic machinery together with a presynaptic diffusion barrier.

\footnotetext{
${ }^{1}$ Leibniz Institut für Molekulare Pharmakologie (FMP) \& Freie Universität Berlin, Department of Molecular Pharmacology and Cell Biology, Robert-RoessleStraße 10, 13125 Berlin, Germany. ${ }^{2}$ Charite Universitätsmedizin, NeuroCure Cluster of Excellence, Virchowweg 6, 10117 Berlin, Germany. ${ }^{3}$ Freie Universität Berlin, Institut für Chemie und Biochemie, Takustrasse 6, 14195 Berlin, Germany. Correspondence and requests for materials should be addressed to J.S. (email: jan.schmoranzer@fu-berlin.de) or to V.H. (email: haucke@fmp-berlin.de).
} 
$\mathrm{D}$ uring synaptic activity non-peptide neurotransmitters are released by exocytic fusion of small clear SVs with the presynaptic membrane at active zones (AZs) followed by compensatory endocytosis of SV membranes from the surrounding periactive zone ${ }^{1-5}$. Although the effect of changes in surface mobility of postsynaptic neurotransmitter receptors on synaptic transmission is well established ${ }^{6-9}$, comparably little is known about the mobility of newly exocytosed SV proteins (NEP) within the presynaptic terminal. This is largely owed to the fact that SV exocytosis operates on a millisecond timescale and is immediately followed by endocytic membrane retrieval, thus, posing technical hurdles to the direct observation of the diffusional behaviour of newly exocytosed SV proteins in primary neurons. Plasma membrane pools of SV proteins (that is, originating from previous rounds of exocytosis) have been shown to either be dispersed within the axonal membrane ${ }^{10}$ or to cluster at presynaptic sites ${ }^{11}$. How these observations relate to the diffusional behaviour of newly exocytosed SV proteins remains unclear though. The fate of newly exocytosed SV proteins likely is of key importance for neurotransmission because the unlimited diffusional escape of SV proteins into the axon would counteract the reformation of properly sized SVs of correct composition by endocytosis. Interestingly, surface-stranded SV proteins have been shown to be preferentially retrieved by endocytosis to regenerate functional SVs ${ }^{12,13}$. Finally, diffusional dispersion of newly exocytosed SV proteins away from AZs may be crucial for the clearance of release sites during sustained high-level activity $^{14,15}$. The diffusional behaviour of newly exocytosed membrane proteins has been assayed previously in fibroblasts ${ }^{16}$ and neuroendocrine PC12 cells ${ }^{16,17}$, which lack proper SVs and AZ-like release sites. Moreover, neuroendocrine cells frequently undergo kiss-and-run exocytosis ${ }^{14,18}$, thereby alleviating the need for a tight coupling between exocytic fusion and endocytic membrane retrieval that is characteristic of neurotransmission in central nervous system neurons ${ }^{1-5}$.

Here, we study the diffusional fate of newly exocytosed SV proteins in primary hippocampal neurons by high-resolution time-lapse imaging. We show that newly exocytosed SV proteins rapidly disperse within the first seconds post fusion until confined within the presynaptic bouton, followed by their slow reclustering. Confinement within the presynaptic bouton but not the rate of reclustering is modulated by SV protein association with the clathrin-based endocytic machinery to limit diffusional spread of newly exocytosed SV proteins. These data suggest that diffusion and axonal escape of newly exocytosed vesicle proteins are counteracted in part by SV protein association with the clathrin-based endocytic machinery and by the presence of a presynaptic diffusion barrier.

\section{Results}

Imaging newly exocytosed SV proteins. We expressed SV proteins lumenally tagged with $\mathrm{pH}$-sensitive pHluorin $\mathrm{GFP}^{19,20}$ in cultured primary hippocampal neurons ${ }^{10,13}$. In resting neurons, the pHluorin signal is quenched because of the low intralumenal $\mathrm{pH}$ (5.5) of SVs, but is dequenched during stimulation-induced exocytosis to the neutral cell exterior ${ }^{19,20}$ (Fig. 1a, Supplementary Fig. 1a). During subsequent membrane retrieval and reacidification pHluorins are requenched (Supplementary Fig. 1a). To exclusively image newly exocytosed SV-pHluorin proteins we capitalized on the observation that SV proteins exocytosed and subsequently endocytosed are nonidentical ${ }^{12,13}$. Selective photobleaching or enzymatic digestion 'eclipsed' the pre-existing surface pool of pHluorintagged synaptobrevin 2 (Syb2) ('surface-eclipsed') ${ }^{13}$ (Fig. 1a,b). When prebleached neurons were stimulated with 40 action potentials (APs), we observed a fast initial rise in fluorescence (F) because of SV exocytosis, followed by a small decrease of $\mathrm{F}$ (by $\sim 15-20 \%$ ) to a plateau level because newly exocytosed SV proteins were not endocytosed and reacidified ${ }^{13}$ (Fig. 1c). As a further confirmation we compared the Syb2-pHluorin responses of prebleached neurons, neurons treated with the vATPase inhibitor folimycin to prevent reacidification ${ }^{21}$, or prebleached folimycin-treated neurons. Under all of these conditions we observed a fast exocytic rise in $\mathrm{F}$ followed by a small decrease of $\mathrm{F}$ to a plateau level (Supplementary Fig. 1b), indicating that the large majority of all newly exocytosed proteins remains at the neuronal surface under these conditions rather than being reinternalized. The small $\mathrm{F}$ decrease at boutons observed post stimulation correlated with an $\mathrm{F}$ increase in the axon, suggesting that a minor fraction of newly exocytosed Syb2 escapes into the axon (Fig. 1d), possibly contributing to sharing of SV proteins between neighbouring boutons ${ }^{22}$. The majority $(\geq 80 \%)$ of newly exocytosed Syb2, however, remained confined within the presynaptic bouton for up to $80 \mathrm{~s}$ (Fig. 1c).

To image the precise spatiotemporal behaviour of newly exocytosed Syb2-pHluorin we combined high-resolution timelapse imaging with synchronized electric field stimulation of surface-eclipsed neurons and semi-automated image analysis. Newly exocytosed pHluorin overlapped with the AZ marker Munc13-1 (Supplementary Fig. 1c) with 151 out of 160 boutons containing a single Munc13-1-positive AZ, suggesting that single synapses were imaged. Signals for newly exocytosed Syb2pHluorin were then determined by fitting of the backgroundand bleach-corrected images $(\Delta \mathrm{F})$ to a two-dimensional (2D) Gaussian using a customized software tool (Fig. 2a). Excellent fits were obtained as demonstrated by the low, noise-like residuals (Fig. 2a, bottom). Hundreds of intensity and full-width halfmaximum $^{2}\left(\mathrm{FWHM}^{2}\right)$ traces recorded from surface-eclipsed neurons were averaged and quantitatively analysed (Fig. 2b,c). FWHM $^{2}$ measured from the Gaussian fits of Syb2-pHluorin increased within the first few seconds of stimulation indicating fast local spread of newly exocytosed SV proteins (Fig. 2a,b), most likely corresponding to free diffusion (see Fig. 3 and below) within the bouton membrane. Diffusional spread within $2 \mathrm{~s}$ post stimulation reached a plateau indicative of the confinement of Syb2-pHluorin in an area of about $2 \mu \mathrm{m}^{2}$, corresponding to the known dimensions of an average presynaptic bouton ${ }^{23}$. The short plateau phase was independent of variable expression levels of Syb2-pHluorin (Supplementary Fig. 1d) and was followed by a slow decrease of $\mathrm{FWHM}^{2}$ that continued for about $80 \mathrm{~s}$ until it reached about $1 \mu \mathrm{m}^{2}$ (Fig. 2c). The observed decrease of FWHM ${ }^{2}$ was not due to photobleaching (Supplementary Fig. 1e), but rather reflected the time-dependent reclustering of exocytosed Syb2, consistent with results from stimulated emission depletion (STED) microscopy of fixed samples ${ }^{11}$. Moreover, diffusional spread, confinement, and reclustering of newly exocytosed SV proteins similar to that seen in photobleached neurons was observed in neurons expressing a tobacco etch virus (TEV)cleavable variant of Syb2-pHluorin following TEV proteasemediated enzymatic digestion of the pre-existing surface pool of Syb2-pHluorin (Supplementary Fig. 1f-h).

Spread, confinement and reclustering of major SV proteins. To probe whether diffusional spread, confinement and slow reclustering were specific properties of exocytosed Syb2 or reflect the general behaviour of all SV proteins we monitored the spatiotemporal dynamics of newly exocytosed pHluorin-tagged synaptophysin (Syp) and synaptotagmin 1 (Syt1), two other major SV proteins. Syt1 and Syp both exhibited diffusional spread within the first seconds of stimulation (Fig. 3a) until confined 
a
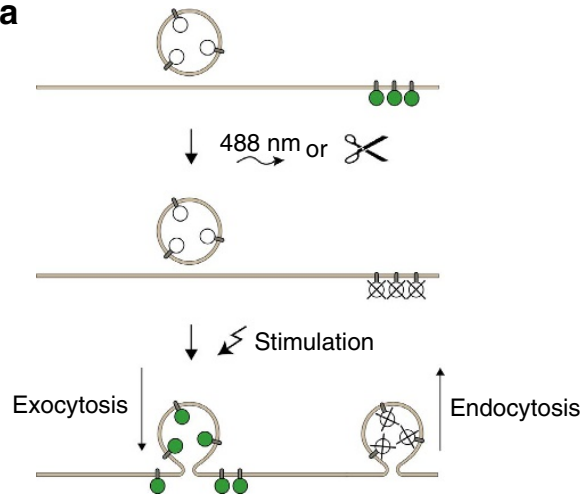

b

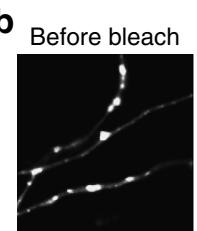

(Enhanced)

After bleach After bleach
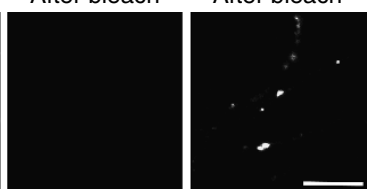

C

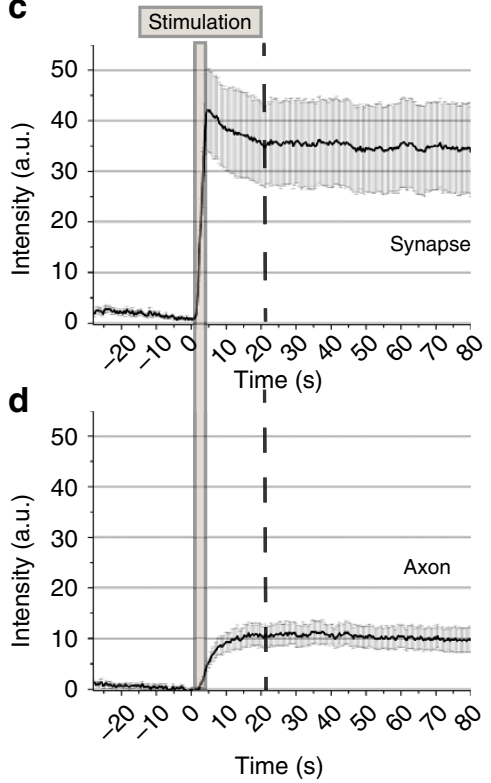

Figure 1 | Newly exocytosed Syb2 is largely retained within presynaptic boutons. (a) Schematic of experimental workflow to monitor newly exocytosed pHluorin-tagged SV proteins. (b) Syb2-pHluorin fluorescence (F) before and after selective photobleaching. Right, enhanced contrast shows small residual $\mathrm{F}$ post-bleaching. Scale bar, $5 \mu \mathrm{m}$. (c,d) Syb2-pHluorin $\mathrm{F}$ intensity changes from time-lapse images $\left(400 \mathrm{~ms}\right.$ frame $\left.{ }^{-1}\right)$ of stimulated $(40 \mathrm{APs}, 20 \mathrm{~Hz})$ surface-eclipsed neurons. (c) Presynaptic boutons, (d) axonal areas (mean \pm s.e.m.; $N=10$ neurons).
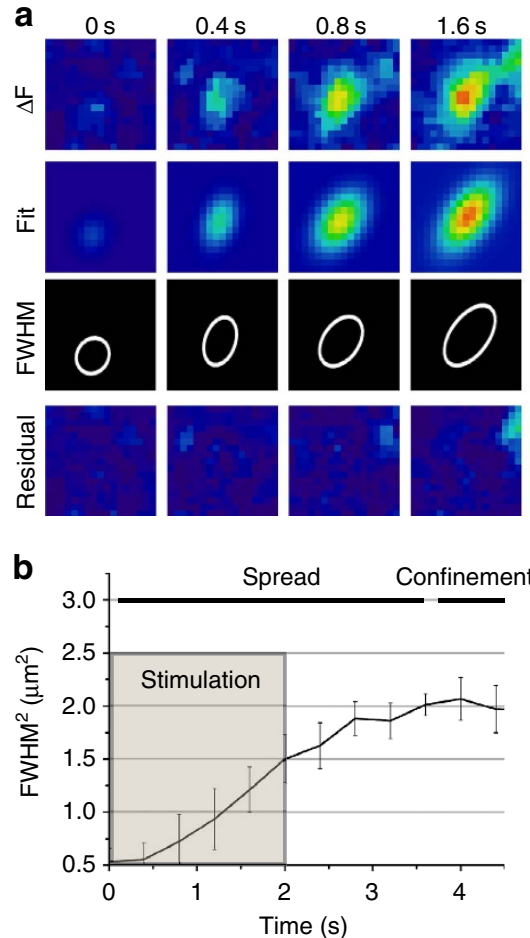
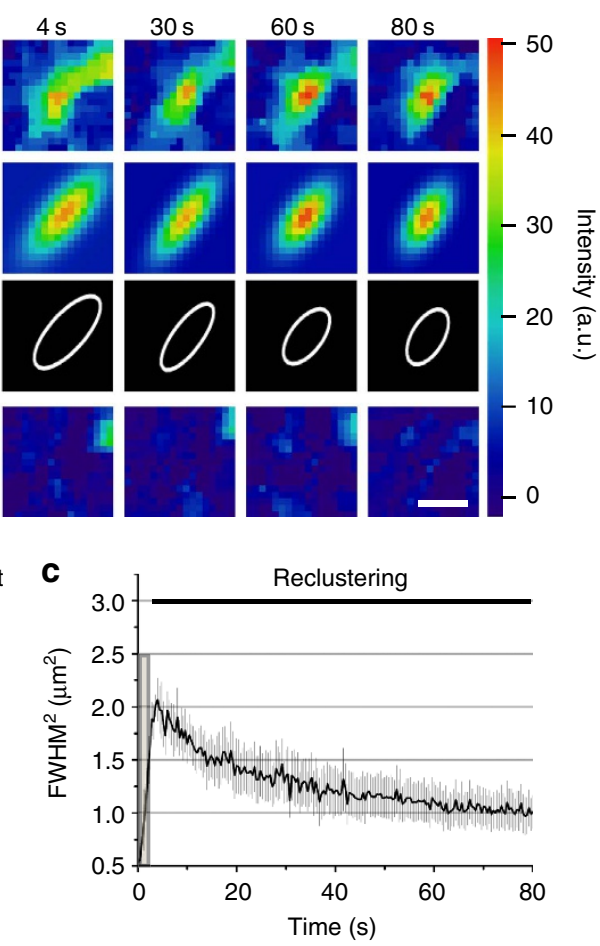

Figure 2 | Syb2 spread, confinement and reclustering within presynaptic boutons. (a) Representative images of a Syb2-pHluorin expressing synapse of stimulated (40 APs, $20 \mathrm{~Hz}$ ) surface-eclipsed neurons, either background subtracted $(\Delta \mathrm{F})$, Gaussian fit (Fit), FWHM (white line) or Gaussian fit residual. Scale bar, $1 \mu \mathrm{m}$. (b,c) FMWH ${ }^{2}$ of Gaussian fit over time. Fast diffusional spread during and after stimulation, followed by confinement (b) and reclustering (c). Mean \pm s.e.m.; $n=5$ independent experiments.

within the $2-2.5 \mu \mathrm{m}^{2}$-sized area of the bouton (Fig. 3a,c), followed by their slow reclustering (Fig. 3b,d) with a $t_{1 / 2}$ of $15-20 \mathrm{~s}$ (Fig. 3e). To test whether the initial spread of newly exocytosed SV proteins was mediated by free diffusion we fed the experimentally detected stimulation-induced increase in Syb2-pHluorin fluorescence (Fig. 3f) into a free diffusion model based on successively fusing SVs. Modelled intensity distributions from single events were summed (Fig. 3g), and the diffusion coefficient was adapted to yield the best-fit between model and experimentally determined $\mathrm{FMWH}^{2}$ for the initial SV protein spread during stimulation (Fig. 3h). This analysis revealed that the initial spread of newly exocytosed SV proteins indeed is described well by free 


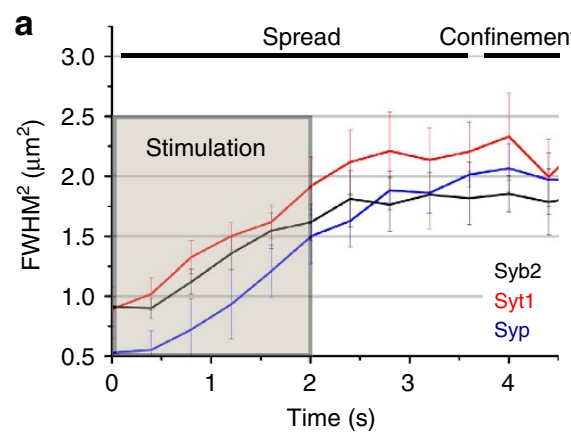

C
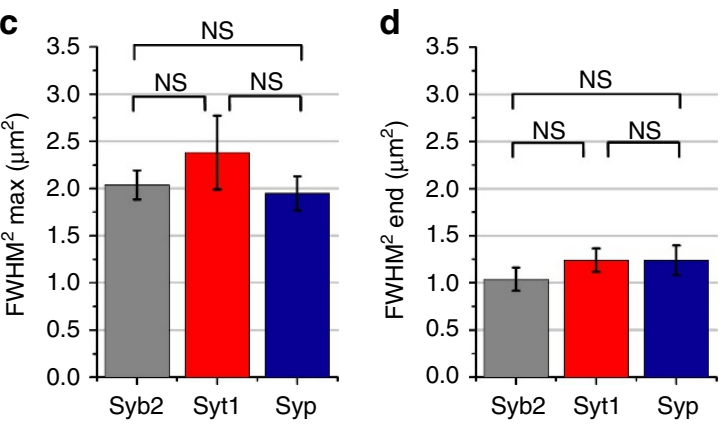

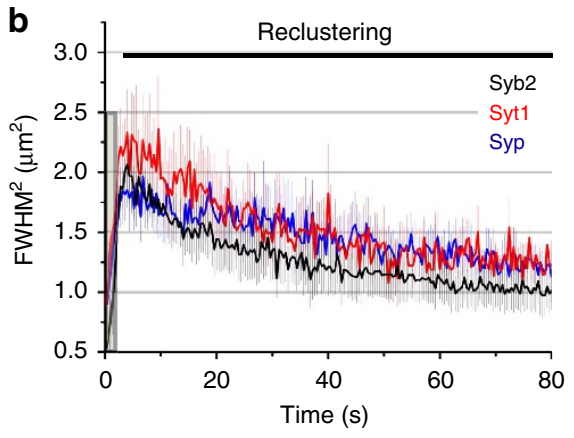

e

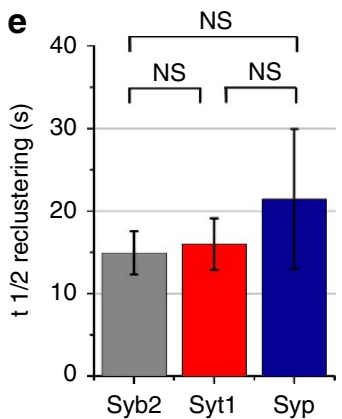

f

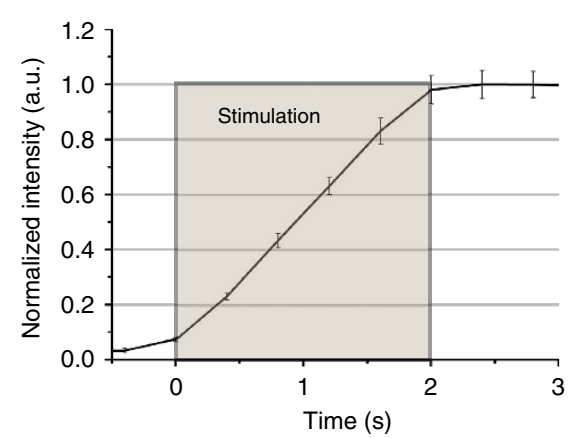

h

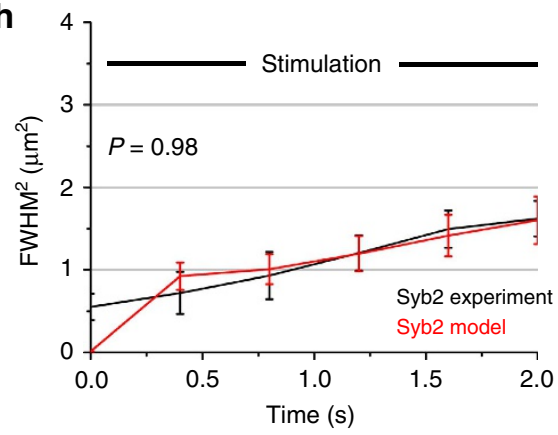

g
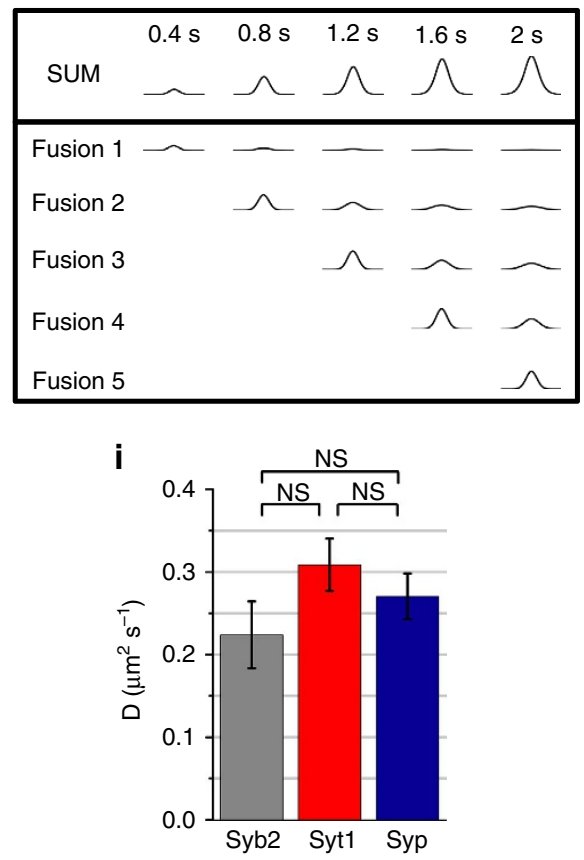

Figure 3 | Diffusional spread, confinement, and reclustering of newly exocytosed SV proteins. (a,b) FMWH ${ }^{2}$ of Gaussian fit over time shows spreading, confinement (a), and reclustering (b) of Syb2-, Syt1- and Syp-pHluorin in surface-eclipsed stimulated (40 AP, $20 \mathrm{~Hz})$ neurons imaged at 400 ms frame ${ }^{-1}$. (c-e) Maximal spread (c), last time point (d) and half-life $t_{1 / 2}$ of reclustering (e). (f) Measured Syb2-pHluorin $F$ increase used to model-free diffusion of successively fusing SVs. (g) Modelled intensity distributions from successive SV fusions and sum distribution. (h) Best-fit of model and experimentally determined FMWH ${ }^{2}$. (i) Diffusion coefficients (D) for Syb2, Syt1, Syp. Mean \pm s.e.m.; independent experiments: Syb2 $(n=5)$, Syt1 $(n=3)$, Syp $(n=3)$. Statistical analysis was done by two-tailed unpaired t-test (c-e, i) or two-way analysis of variance with repeated measures ( $h$ time points $0.8-2 \mathrm{~s}$ ). NS: $P>0.05$.

diffusion within the first few seconds. We obtained similar diffusion coefficients for the initial dispersion of Syb2, Syt1 and Syp of $\sim 0.20-0.35 \mu \mathrm{m}^{2} \mathrm{~s}^{-1}$ (Fig. 3i; Supplementary Fig. 2a,b), a value resembling that reported for mobile postsynaptic AMPA receptors ${ }^{24}$. This value would translate into a movement of SV proteins away from AZs at a speed of $\sim 1 \mu \mathrm{m}$ in $2 \mathrm{~s}$, explaining the need for confinement of newly exocytosed SV proteins within presynaptic boutons to prevent their substantial loss into axons.
The endocytic machinery limits spread and confinement. As surface-stranded SV proteins form a reservoir for endocytic membrane retrieval ${ }^{12}$, we hypothesized that association of SV proteins with the endocytic machinery contributes to their presynaptic confinement. To test this, we analysed the postexocytic diffusional behaviour of Syb2 (M46A), a missorted Syb2 mutant ${ }^{25}$ that selectively fails to associate with the Syb2-specific endocytic adaptors AP180 and CALM ${ }^{26,27}$ resulting in its partial 
redistribution $(\sim 70 \%$ of total $)$ to the plasma membrane ${ }^{26}$ (Supplementary Fig. 3a). Surface-eclipsing of the large surfacestranded pool of Syb2 (M46A)-pHluorin by enzymatic TEV protease-mediated digestion (Supplementary Fig. 3b) allowed us to nonetheless reliably track its diffusional fate, albeit with reduced signal-to-noise ratio. Syb2 (M46A) displayed rapid diffusional dispersion, confinement and slow reclustering similar to wild-type (WT) Syb2 (Fig. 4a,b). However, diffusing M46A explored a significantly larger area than WT Syb2 (Fig. $4 \mathrm{a}-\mathrm{c}$ ), and this difference in the $\mathrm{FMWH}^{2}$ remained after M46A had undergone slow reclustering (Fig. $4 \mathrm{~d}$ ), while $t_{1 / 2}$ of reclustering was largely unaffected in the mutant (Fig. 4e). Furthermore, kinetic mathematical modelling of our experimental data revealed a significantly increased initial rate of diffusion of M46A compared with WT Syb2 (Fig. 4f-h).

To further investigate the contribution of the endocytic machinery to presynaptic confinement of newly exocytosed Syb2, we eliminated neuronal expression of the Syb2-specific endocytic adaptor proteins CALM and AP180 (refs 26,27). When WT Syb2-pHluorin was expressed in neurons lacking AP180 (Supplementary Fig. 3c) and depleted of CALM by specific shRNA $^{28}$ (Supplementary Fig. 3d), newly exocytosed pHluorin molecules explored a significantly larger area compared with WT neurons (Fig. $5 \mathrm{a}-\mathrm{c}$ ), and this difference in the $\mathrm{FMWH}^{2}$ remained after Syb2-pHluorin had undergone slow reclustering (Fig. 5d), while $t_{1 / 2}$ of reclustering was largely unaffected (Fig. 5e), thus phenocopying the effects of Syb2 (M46A). We conclude that sustained abrogation of Syb2 association with its endocytic adaptors AP180 and CALM either by mutation of Syb2 (that is, M46A) or elimination of AP180/CALM expression modulates its diffusional spread and confinement. Consistent with these data we observed that acute perturbation of the endocytic machinery by the clathrin inhibitor Pitstop 2 (ref. 29; see also refs 30-32) also led to a similar increase in the area of Syb2 confinement (Supplementary Fig. 3e-h).

Collectively, these data suggest that diffusional spread and confinement of newly exocytosed Syb2 are limited at least in part by its association with endocytic proteins. To investigate this hypothesis further we analysed the localization and dynamics of the clathrin-based endocytic machinery. Live imaging of hippocampal neurons co-expressing Munc13-1-mCherry or Syb2-pHluorin and fluorescent protein-tagged clathrin light chains (LC) showed clathrin to be concentrated in presynaptic boutons, where it localizes at and around AZs (Supplementary Fig. 4a,b), in close proximity to newly exocytosed SV proteins (Supplementary Fig. 4c,d). The distribution of eGFP-clathrin LC or mRFP-clathrin LC following stimulation of neurons with 40 APs remained grossly unchanged (Supplementary Fig. 4b,d). To more precisely determine the sub-synaptic localization of endocytic proteins and their spatial relationship with the pool of newly exocytosed SV proteins we turned to multicolour superresolution microscopy. We first used three-dimensional (3D) Structured Illumination Microscopy (3D-SIM), a technique that provides an approximately 2 -fold gain in both lateral $(x y)$ and a
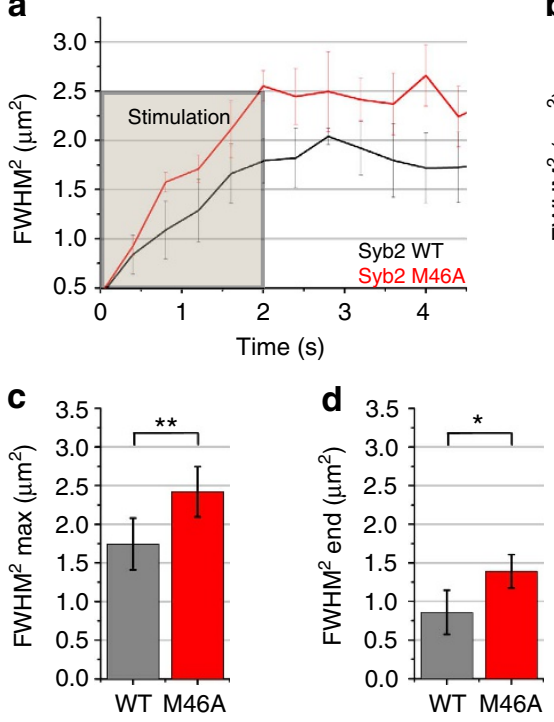

b
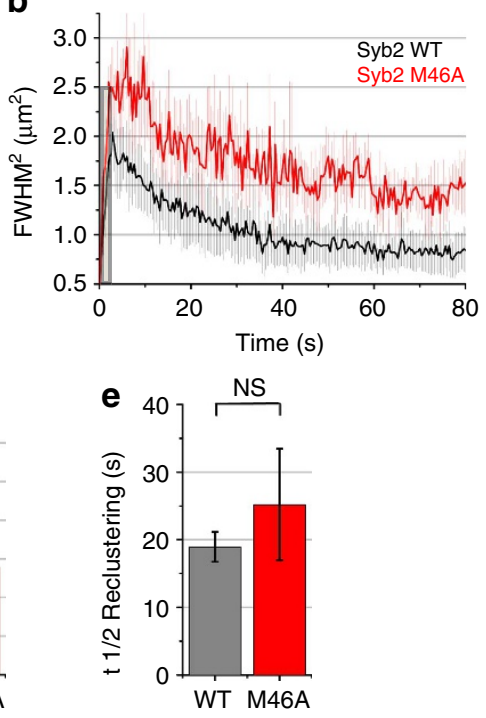

$\mathbf{f}$

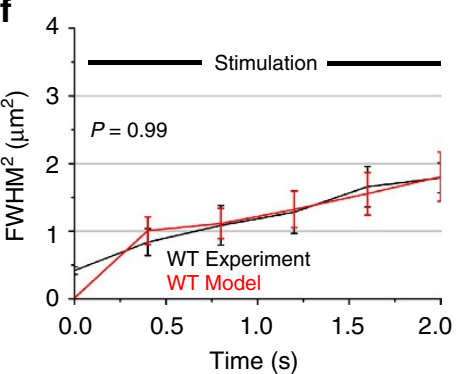

g

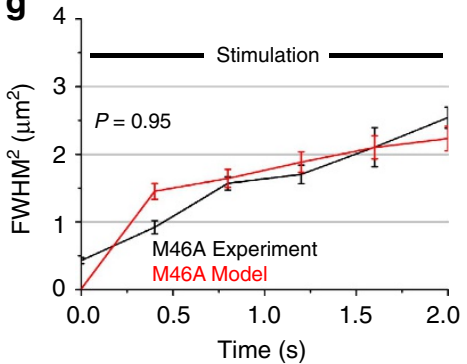

h

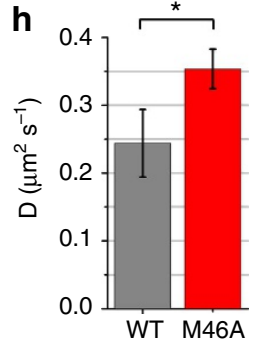

Figure 4 | Spread and confinement of Syb2 are modulated by its association with AP180 or CALM. (a,b) FMWH² of Gaussian fit over time shows spreading, confinement (a), and reclustering (b) of Syb2-pHluorin WT or M46A in surface-eclipsed stimulated (40 AP, $20 \mathrm{~Hz})$ neurons imaged at 400 ms frame $^{-1}$. (c-e) Maximal spread (c), last time point (d) and half-life $t_{1 / 2}$ of reclustering $(\mathbf{e})$. (f,g) Best-fit of model and experimentally determined $\mathrm{FMWH}^{2}$ for Syb2 WT and M46A. (h) Diffusion coefficients for Syb2 WT and M46A. Mean \pm s.e.m.; $n=3$ independent experiments. Statistical analysis was done by two-tailed paired $t$-test $(\mathbf{c}-\mathbf{e}, \mathbf{h})$ or two-way analysis of variance with repeated measures $(\mathbf{f}, \mathbf{g})$ time points $0.8-2 \mathrm{~s}) .{ }^{\star \star} P<0.01,{ }^{\star} P<0.05$,

NS: $P>0.05$ 

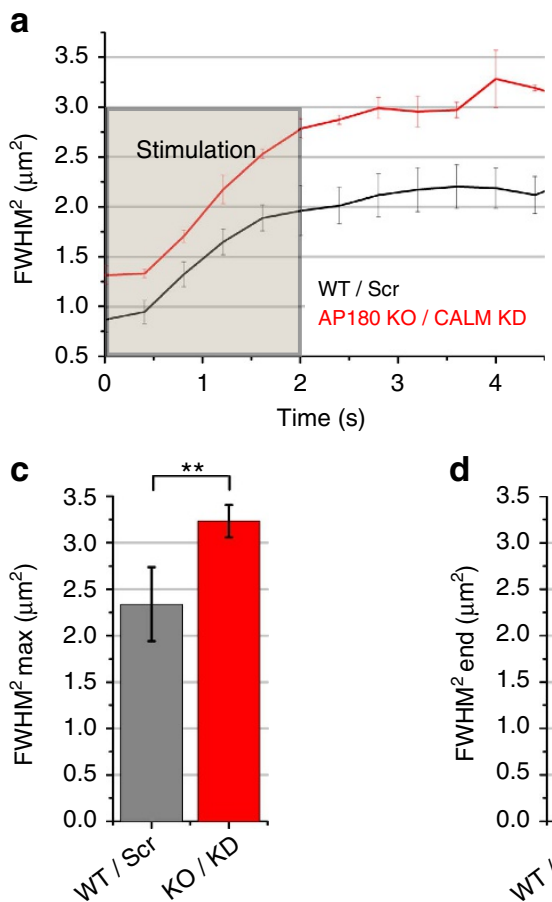

d
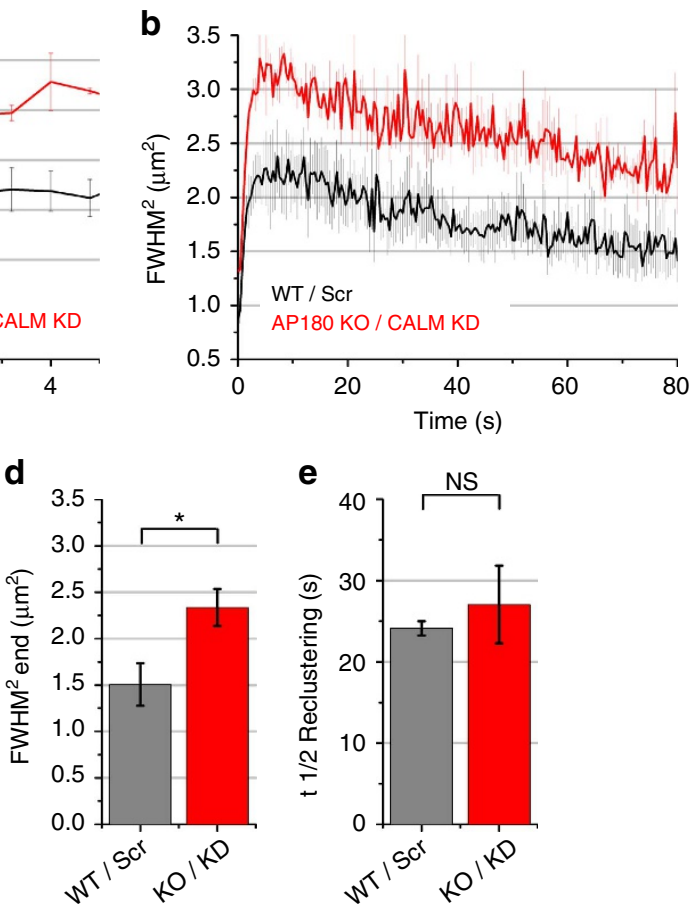

Figure 5 | AP180 and CALM modulate spread and confinement of newly exocytosed Syb2. (a,b) FMWH² of Gaussian fit over time shows spreading, confinement (a) and reclustering (b) of Syb2-pHluorin in surface-eclipsed hippocampal neurons lacking AP180 (KO) and depleted of CALM (knockdown $(\mathrm{KD})$ ) or corresponding WT control neurons treated with scrambled ( $\mathrm{Scr}$ ) shRNA (stimulation: $40 \mathrm{AP}, 20 \mathrm{~Hz}$ ). (c-e) Maximal spread (c), last time point (d) and half-life $t_{1 / 2}$ of reclustering (e). Mean \pm s.e.m.; $n=3$ independent experiments). Statistical analysis was done by two-tailed paired $t$-test. ${ }^{\star} \times P<0.01,{ }^{\star} P<0.05, \mathrm{NS}: P>0.05$.

axial $(z)$ resolution compared with conventional confocal imaging and allows the use of conventional fluorophores. Multicolour 3D-SIM analysis of hippocampal neurons revealed clathrin and AP180 to localize around the AZ labelled with Bassoon (Fig. 6a), and this distribution remained largely unaffected by stimulation of neurons with $40 \mathrm{APs}$, consistent with live imaging (Supplementary Fig. 4a-d). Finally, to determine the exact spatial relationship between the endocytic machinery and newly exocytosed SV proteins we used spectral demixing direct stochastic optical reconstruction microscopy (SD-dSTORM $)^{33}$, a dSTORM method that allows registration-free dual-colour imaging (Supplementary Fig. 4g) with a lateral resolution of 20-35 nm (refs 33,34). Dual-colour SD-dSTORM analysis of hippocampal synapses stimulated with 40 APs revealed newly exocytosed Syb2 molecules (Supplementary Fig. 4e,f) to cluster around the AZ labelled by Bassoon (Fig. 6c). Both, AP180 and newly exocytosed Syb2 formed sub-synaptic clusters (Fig. 6d, Supplementary Fig. 4h), which partially colocalized, indicated by an overlap of both maxima in the radial intensity profiles on subsynaptic clusters (Fig. 6e) and by the peak in the $k$-nearest neighbour analysis $(\sim 20-30 \mathrm{~nm})$ that lies below the cluster size and within the range of the experimental resolution (Fig. 6f $)^{34}$. The sub-synaptic NEP clusters had average radii of $\sim 50 \mathrm{~nm}$ (Fig. 6e), consistent with a distribution peak at distances of $100 \mathrm{~nm}$ (Fig. 6d). Interestingly, the surface area of these $100 \mathrm{~nm}$ flat clusters is similar to the surface area of native spherical SVs in brain with a diameter of $\sim 40-45 \mathrm{~nm}$ (ref. 35). This suggests that newly exocytosed SV protein clusters likely correspond to the readily retrievable pool of SV proteins, which following endocytosis gives rise to SVs. We conclude that confinement of SV proteins within presynaptic boutons is effected in part by their association with components of the endocytic machinery.

\section{Discussion}

Our data based on high-resolution imaging and Gaussian fit analysis show that newly exocytosed SV proteins following an initial short phase of free diffusion are confined within presynaptic boutons, a process aided by their association with the endocytic machinery (Figs 4-6), in agreement with earlier studies in Drosophila employing an acute block of endocytosis in dynamin/shibire ts $^{\text {mutants }}{ }^{36}$. However, given the moderate effect of abolishing association of Syb2 with AP180 and CALM by mutation or by depleting neurons of both endocytic proteins, it is likely that escape of newly exocytosed SV proteins from the boutons into the axon is further limited by the presence of a presynaptic diffusion barrier, the identity of which remains unknown. We therefore suggest a model according to which newly exocytosed SV proteins diffusionally spread within the bouton, where they are captured by endocytic adaptors (e.g. AP180 and CALM for Syb2) localized around AZ release sites (Figs 6,7) 37,38 . Endocytic SV protein association in this model, thus, not only aids sorting of SV proteins, but also restricts diffusional escape of newly exocytosed vesicle proteins into the surrounding axon. This model is also consistent with recent data from neuromuscular junctions, where the pool of actively recycling SVs has been shown to be kept largely separate from the reserve pool $^{39}$ and with super-resolution imaging of the mesoscale organization of exocytic membrane proteins in neuroendocrine PC12 cells, where they form patches ${ }^{40}$ similar in size to newly exocytosed SV protein clusters observed here (see Fig. 6).

Diffusional spread and confinement of newly exocytosed SV proteins is incompatible with kiss-and-run SV exo-/endocytosis in central nervous system neurons, in which case exocytosed SV proteins would remain confined to the site of fusion 
a

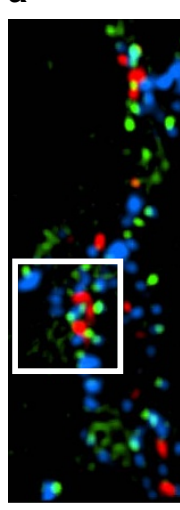

b

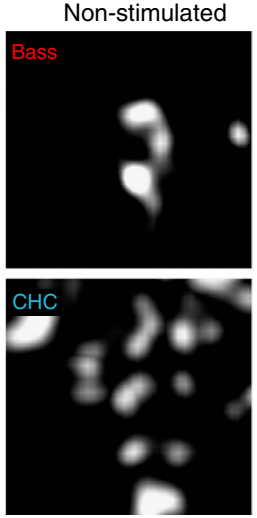

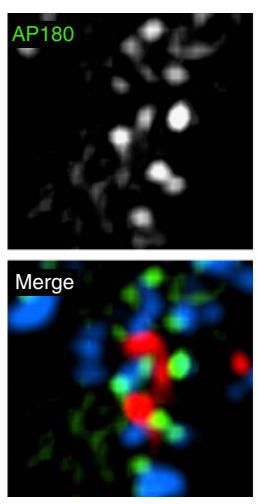

Stimulated
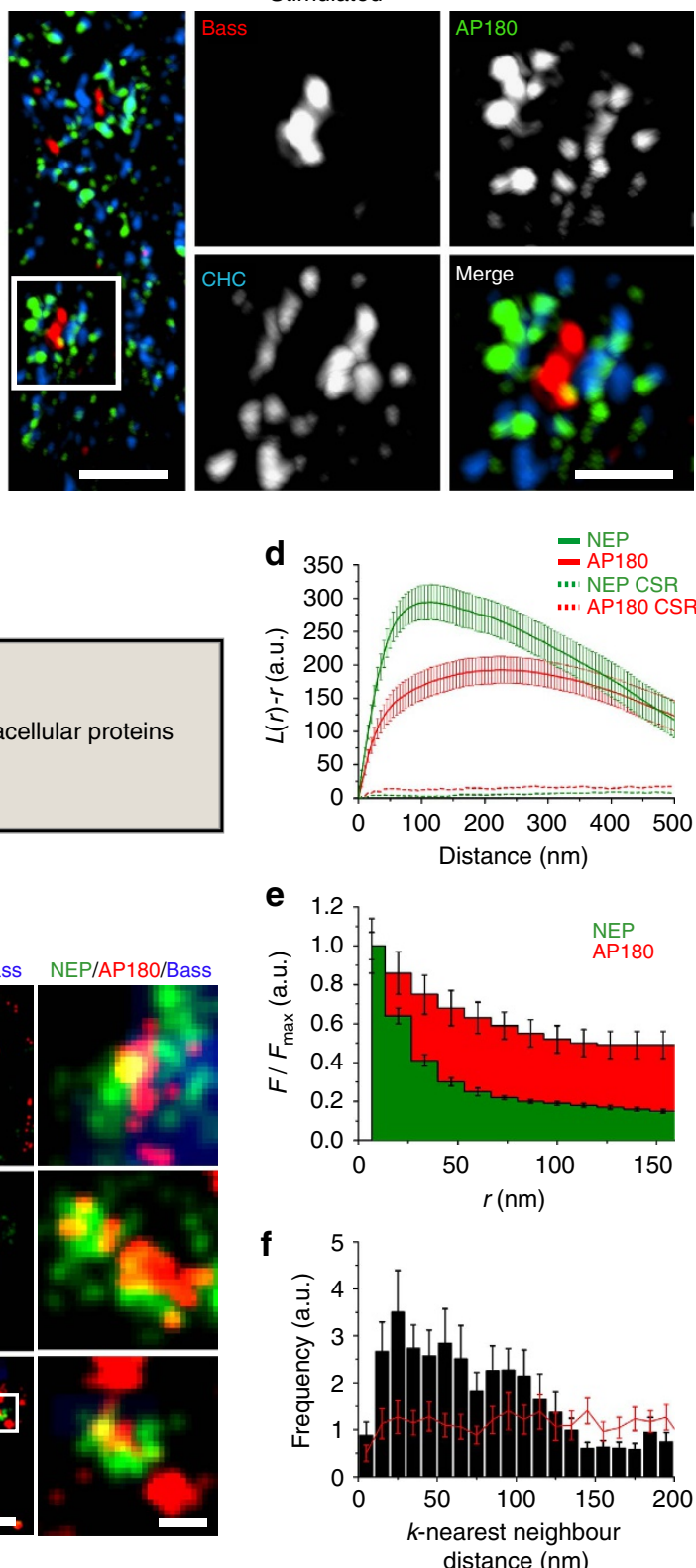

Figure 6 | Newly exocytosed proteins co-cluster with endocytic proteins in the periactive zone. (a) 3D-Structured Illumination Microscopy (3D-SIM) analysis of Bassoon (Bass), AP180 and clathrin heavy chain (CHC) in stimulated (40 APs, $20 \mathrm{~Hz}$ ) or non-stimulated hippocampal neurons. Scale bar, $1 \mu$ m; zoom, $500 \mathrm{~nm}$. (b) Schematic for immunolabeling of the newly exocytosed SV protein pool (NEP). (c-e) SD-dSTORM imaging of newly exocytosed SV proteins (stimulation: $40 \mathrm{AP}, 20 \mathrm{~Hz}$ ) in hippocampal neurons expressing Syb2-c-myc. (c) 2-colour SD-dSTORM analysis of NEP and AP180, overlaid with wide-field image of Bassoon. Scale bar, $1 \mu \mathrm{m} ; \mathrm{zoom}, 100 \mathrm{~nm}$. (d) Ripley's $L(r)-r$ function shows sub-synaptic clustering of NEP and of AP180, indicated by positive $L(r)-r$ values with a maximum at radii of about $100 \mathrm{~nm}$ (NEP) and $200 \mathrm{~nm}$ (AP180). Mean \pm s.e.m.; $n=31$ synapses. Dotted lines represent upper envelopes of complete spatial randomness (CSR). (e) Radial intensity profiles of NEP and AP180 signals centred on the maxima of sub-synaptic NEP clusters show colocalization of both proteins (mean \pm s.e.m.; $n=47$ synapses). ( $\mathbf{f}$ ) $k$-Nearest neighbour analysis $(k=10)$ of NEP and AP180 showing a maximum at $25 \mathrm{~nm}$. The red line represents results after toroidal shift of one channel. Values above the red line indicate colocalization of NEP and AP180 (mean \pm s.e.m.; $n=31$ synapses).

for immediate retrieval ${ }^{41,42}$ without intermixing with plasma membrane components ${ }^{10}$. Similar observations have been reported for the exocytosis and rapid recapture of vesicular acetylcholine transporter in PC12 cells ${ }^{17}$, although in this case it has remained unclear to what extent recapture may reflect clathrin-mediated endocytosis of the transporter.

Following confinement, we observe slow reclustering of exocytosed SV proteins within the periactive zone. The extent but not the rate of reclustering depends on association of SV proteins with the endocytic machinery (Figs 4,5), suggesting the involvement of additional factors. These may include interactions between SV proteins themselves ${ }^{43-45}$ and/or of SV proteins with membrane lipids such as cholesterol ${ }^{46}$, or components of the cytoskeleton. Interestingly, treatment of neurons with latrunculin A did not affect SV protein diffusion or reclustering in our hands (Supplementary Fig. 5). Hence, filamentous actin is unlikely to 


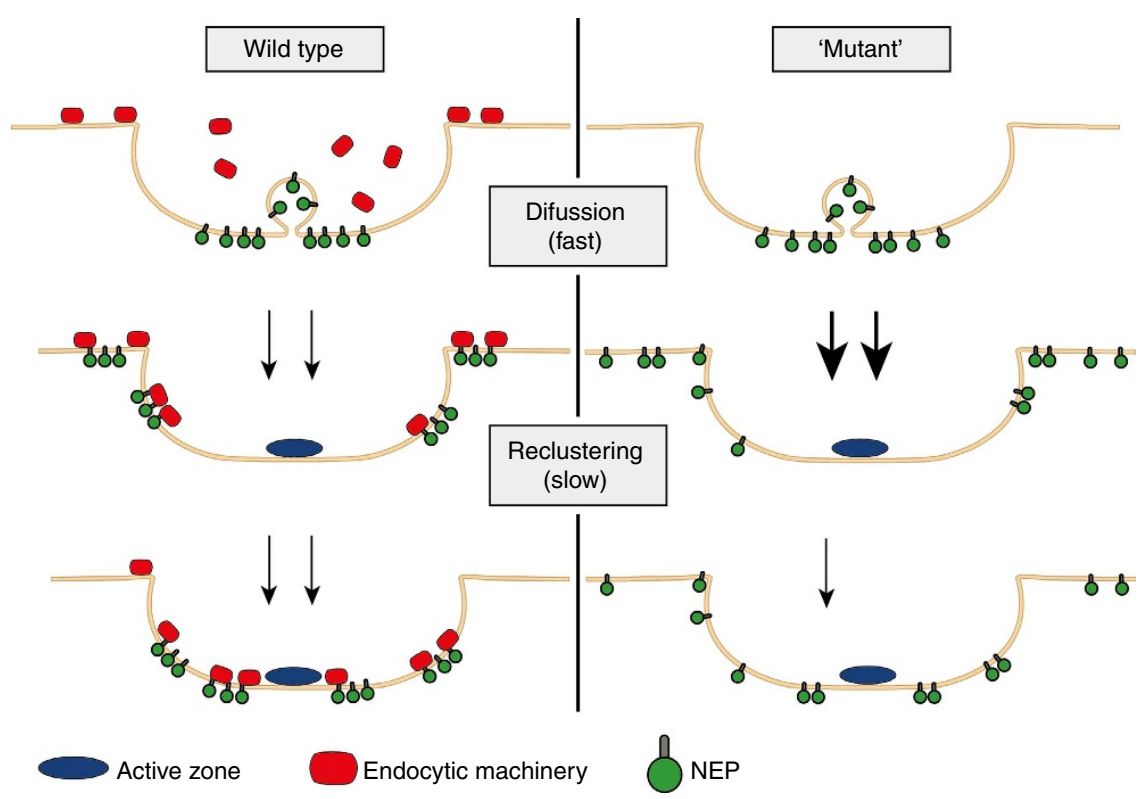

Figure 7 | Model of protein diffusion, confinement and reclustering. Schematic model of SV protein confinement and reclustering and the role of the endocytic machinery: following rapid diffusion (WT, top) newly exocytosed SV proteins remain confined within the presynaptic bouton (WT, middle), aided by their association with the endocytic machinery. Confinement is followed by SV protein reclustering within the periactive zone (WT, bottom). In the absence of a functional endocytic machinery diffusion of newly exocytosed SV proteins is accelerated (bold arrows) and both confinement and the extent, but not the rate, of reclustering are compromised.

play an essential role in these processes, although it remains possible that local actin pools in presynaptic boutons are resistant to the drug or that short actin filaments or formin-dependent actin hotspots ${ }^{47}$ suffice to prevent SV protein diffusion and/or to facilitate reclustering. Such reclustered SV proteins likely constitute the readily retrievable pool of $\mathrm{SVs}^{12}$ that may subsequently be internalized by clathrin-dependent ${ }^{48}$ or bulk endocytosis mechanisms including ultrafast endocytosis ${ }^{49,50}$. We therefore propose that diffusional spread, confinement, and reclustering of SV proteins represent key steps in the SV exo-endocytic cycle that underlies neurotransmission.

\section{Methods}

Neuronal cell cultures and transfection. Primary hippocampal neuron cultures were prepared from mouse brain (P0-P3) and transfected at 7-8 days in vitro (DIV) by a modified calcium phosphate transfection protocol (Promega). Neurons were imaged at 14-15 DIV.

Live cell imaging of hippocampal neurons. Images were acquired using an inverted fluorescence microscope (Eclipse Ti, Nikon), controlled by MicroManager 4.11 (ref. 51), equipped with a $\times 60$ oil-immersion objective $(N A=1.49$, Nikon), a sCMOS camera (Neo, Andor) and a 200 Watt mercury lamp (Lumen 200, Prior). Images were acquired at $2.5,0.5$ or $0.2 \mathrm{~Hz}$ with the corresponding filter set (EGFP/pHluorin: F36-526, mCherry/mRFP: F36-504, AHF Analysentechnik). Imaging was performed at $30^{\circ} \mathrm{C}$ in physiological imaging buffer $(170 \mathrm{mM} \mathrm{NaCl}$,

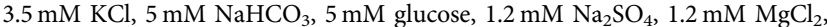
$1.3 \mathrm{mM} \mathrm{CaCl}_{2}, 0.4 \mathrm{mM} \mathrm{KH}_{2} \mathrm{PO}_{4}, 20 \mathrm{mM}$ TES (2-[(2-Hydroxy-1,1-

bis(hydroxymethyl)ethyl)amino] ethanesulfonic acid), pH 7.4). $50 \mu \mathrm{M}$ APV (DL-2-Amino-5-phosphonopentanoic acid, Sigma-Aldrich) and $10 \mu \mathrm{M}$ CNQX (6-Cyano-7-nitroquinoxaline-2,3-dione, Sigma-Aldrich) were added to the imaging buffer to silence spontaneous neurotransmission. Unquenched surface pHluorin was either selectively bleached by illuminating the neurons for $400 \mathrm{~s}$ prior to stimulation or the pHluorin tag was removed by 5 min enzymatic digestion with $4 \mu \mathrm{g} \mathrm{ml}^{-1}$ TEV protease in imaging buffer. Both procedures were sufficient to eclipse fluorescence signal from the surface pool of SV proteins such that any change in pHluorin signals after stimulation originated only from newly exocytosed protein, but not from pre-existing surface pools. Neurons were stimulated by electric field stimulation with $40 \mathrm{APs}$ (at $20 \mathrm{~Hz}, 100 \mathrm{~mA}$ ) in a stimulation chamber (RC-47FSLP, Warner Instruments). In Supplementary Fig. 1b reacidification was blocked by treating neurons for $1 \mathrm{~min}$ with $67 \mathrm{nM}$ folimycin (in ethanol, $1 \mu \mathrm{ml}^{-1}$ imaging buffer). For experiments shown in Supplementary
Fig. 3e-g, hippocampal neurons were treated with DMSO $\left(0.67 \mu \mathrm{ml}^{-1}\right.$ imaging buffer) and stimulation-induced pHluorin responses were recorded. Neurons were then treated for $2 \mathrm{~min}$ with $20 \mu \mathrm{M}$ Pitstop 2 (in DMSO, $0.67 \mu \mathrm{ml}^{-1}$ imaging buffer) before stimulation to fill endocytic slots with SV cargo. Following a second round of surface-bleaching stimulation-induced pHluorin responses were recorded. For experiments shown in Supplementary Fig. 5a,b hippocampal neurons were treated for 5 min with $10 \mu \mathrm{M}$ Latrunculin A (in DMSO, $1 \mu \mathrm{ml}^{-1}$ imaging buffer) or DMSO before stimulation. For the experiments shown in Supplementary Fig. 5c,d hippocampal neurons were transfected with LifeAct-eGFP. Images were taken before (DMSO, $1 \mu \mathrm{ml}^{-1}$ imaging buffer) and after $5 \mathrm{~min}$ of treatment with $10 \mu \mathrm{M}$ Latrunculin A (in DMSO, $1 \mu \mathrm{ml}^{-1}$ imaging buffer).

Image analysis. Identification of responding synapses: synaptic areas displaying stimulation-induced pHluorin responses were identified based on stimulus-evoked changes in local fluorescence signal (F) using a custom-written ImageJ macro ${ }^{52}$. The synaptic area was calculated from the differential image $\left(F_{\text {after }}-F_{\text {before }}\right)$ based on a histogram-based threshold procedure.

Correction for photobleaching and background: Regions of interest containing responding synapses were extracted and intensity traces were fit with an exponential decay (Equation. 1) for each single pixel until the start of stimulation.

Exponential decay:

$$
I(t)=b g+a \cdot \mathrm{e}^{-\tau \cdot t}
$$

$I(t)$ : Intensity at time $t ; b g$ : background signal; $a$ : intensity at time $0 ; \tau$ : lifetime The bleach rate was calculated from the lifetime of the pHluorin decay for each pixel (Equation. 2)

Bleach rate:

$$
\lambda=\mathrm{e}^{-\tau}
$$

$\lambda$ : bleach rate; $\tau$ : lifetime

The bleach rate was used to correct the pixel-specific bleaching over the entire image sequence (Equation. 3).

Bleach correction:

$$
I_{\mathrm{bc}}(t)=I(t)+\sum_{i=0}^{t} \lambda \cdot\left(I(i)-I_{0}\right)
$$

$I_{\mathrm{bc}}(t)$ : bleach corrected intensity at time $t ; I(t)$ : Intensity at time $t ; \lambda$ : bleach rate; $I_{0}$ : intensity at time 0 .

To reduce background and to correct for the residual fluorescence signal after photobleaching or TEV treatment (from partially quenched luminal pHluorin molecules), we subtracted the median of five images before stimulation from the entire image sequence resulting in $\Delta \mathrm{F}$ (Fig. 2a).

Quantification of pHluorin intensity signal and escape: the pHluorin intensity signal over time was measured from selected (responding) synapses (see above). The escape of newly exocytosed SV proteins into the axon was quantified by 
manually selecting axonal regions of $0.9 \mu \mathrm{m}^{2}$ with a distance of $1.2 \mu \mathrm{m}$ to a responding synapse.

Quantification of local protein spread by Gaussian fit: The bleach and background corrected image sequences of responding synapses were fit frame-byframe to a 2D Gaussian distribution using a custom-written Igor Pro macro (Equations 4-6). The maximum $\mathrm{FWHM}^{2}$ was analysed over time (Equation 7).

Gaussian fit 2D:

$$
\begin{gathered}
I(x, y)=I_{0}+A \cdot \mathrm{e}^{-\frac{1}{2}\left(\frac{x^{\prime}}{\sigma_{x}}\right)^{2}-\frac{1}{2}\left(\frac{y^{\prime}}{\sigma_{y}}\right)^{2}} \\
x^{\prime}=x \cdot \cos (\theta)+y \cdot \sin (\theta)-x_{0} \cdot \cos (\theta)-y_{0} \cdot \sin (\theta) \\
y^{\prime}=-x \cdot \sin (\theta)+y \cdot \cos (\theta)+x_{0} \cdot \sin (\theta)-y_{0} \cdot \cos (\theta) \\
F W H M=2 \cdot \sqrt{2 \cdot \ln (2)} \cdot \sigma
\end{gathered}
$$

$I(x, y)$ : pixel intensity at the image coordinates $x, y ; I_{0}$ : intensity offset; $A$ : amplitude; $\sigma$ : s.d.; $\theta$ : rotation angle; FWHM: full-width at half-maximum intensity; $x_{0}, y_{0}$ : Gaussian centre.

Diffraction-limited rendering of SD-dSTORM images: to compare the SD-dSTORM images with images of conventional wide-field resolution the SD-dSTORM images were blurred with a Gaussian in ImageJ using $\sigma_{\text {Render }}$ from Equation 8.

Rendering:

$$
\sigma_{\text {Widefield }}=\sqrt{\sigma_{\text {dSTORM }^{2}}+\sigma_{\text {Render }^{2}}}
$$

$\sigma_{\text {Widefield: }}$ standard deviation of wide-field image (Equations 7,9 ); $\sigma_{\text {dSTORM: }}$ standard deviation of SD-dSTORM ${ }^{34} ; \sigma_{\text {Render }}$ standard deviation for rendering. Wide-field resolution:

$$
F W H M=\frac{\lambda}{2 N A}
$$

FWHM: full-width at half-maximum intensity; $\lambda$ : emission wavelength of the dye; NA: numerical aperture of the objective

Finally, the blurred images were displayed with the camera pixel size of $105 \mathrm{~nm}$.

Ripley's $L(r)-r$ function (Fig. 6d): Estimation of normalized and edge-corrected Ripley's $K(r)$ and $L(r)-r$ function (Equations 10,11) were calculated using the splancs package for the $\mathrm{R}$ software environment for statistical computing ${ }^{53}$ using the following equations ${ }^{54}$ :

Ripley's:

$$
\begin{gathered}
K(r)=\frac{A}{N} \sum_{i} \sum_{j \neq i} \frac{I\left(d_{i j}<r\right)}{N \cdot w\left(I_{i}, I_{j}\right)} \\
L(r)-r=\sqrt{\frac{K(r)}{\pi}}-r
\end{gathered}
$$

$K(r)$ : edge-corrected Ripley's $K$ to estimate the number of individual localizations (i) within a distance $\mathrm{r}$ of another $(j)$ localization normalized by the number of total localizations $N$ per area $A ; d_{i j}$ is the distance between localization $i$ and $j ; I\left(d_{i j}<r\right)$ is the indicator function that equals 1 if $d_{i j}<r$ or 0 otherwise; $w\left(l_{i}, l_{j}\right)$ is the weight function for edge correction.

$L(r)-r$ : normalized $K(r)$ equals 0 for a random distribution ${ }^{55}$. Dotted lines (Fig. 6d) represent upper envelopes from 10 simulations of complete spatial randomness (CSR) for each region of interest.

$K$-nearest neighbour: The $k$-nearest neighbour distribution was calculated from localizations within synaptic region of interests in SD-dSTORM images, using a custom-written Image J macro ${ }^{52}$. $K=10$ was chosen in order to reduce the likelihood of false positive hits due to noise. The negative control for colocalization was generated by performing a toroidal shift of one channel for half a region of interest size against the other channel along the $x$ axis.

Radial intensity profiles: Radial intensity profiles were generated with the ImageJ macro 52 'radial profile'. Local maxima were found automatically with the ImageJ function 'find maxima'. Averaged intensity values were plotted against their radial distance to a local maximum in the reference channel (newly exocytosed Syb2).

Modelling diffusional spread of successive SV fusions. To determine diffusion coefficients of pHluorin-tagged synaptobrevin 2 (Syb2), synaptotagmin 1 (Syt1) and synaptophysin (Syp), we assumed a model of successively fusing SVs during stimulation. By using the experimentally determined intensity traces we calculated the relative number of fused SVs at each time point during stimulation. Assuming that the spread of the SV proteins is governed by Brownian motion (free diffusion) each vesicle was treated as an individual Gaussian intensity distribution that was added to the previous intensities. Each Gaussian distribution was weighted by its corresponding intensity increase (experimental data) and integrated with the previous Gaussian intensity distributions (Equations 12,13). The sum of all distributions was fit with another Gaussian (Equation 14) and plotted as the 'model' in Figs 3h,4f,g and Supplementary Fig. 2. The diffusion coefficients were determined by matching the mean slope from the experimental $\mathrm{FWHM}^{2}$ to the FWHM $^{2}$ from the model. The validity of the model was tested by comparing traces obtained from the model and experimentally obtained traces, using a two-way analysis of variance with repeated measures.

Integrated Gaussian:

$$
I(r, t)=\sum_{j=0}^{N} \frac{I_{j}-I_{j-1}}{4 \cdot \pi \cdot D \cdot(n-j)} \cdot \mathrm{e}^{\left(-\frac{r^{2}}{4 \cdot D \cdot(n-j)}\right)}
$$

$$
N=\frac{t}{f}
$$

$I(r, t)$ : intensity at the radial distance $r$ (from Gaussian center) and time $t$;

$D$ : diffusion coefficient; $n$ : frame number; $f$ : frame duration.

Gaussian Fit 1D:

$$
I(r)=I_{0}+A \cdot \mathrm{e}^{-\frac{1}{2}\left(\frac{r}{\sigma}\right)^{2}}
$$

$I(r)$ : intensity at the radial distance $r$ (from Gaussian centre); $I_{0}$ : intensity offset; $\sigma$ : s.d.

Immunocytochemistry. Primary hippocampal neurons were fixed with $4 \%(\mathrm{w} / \mathrm{v})$ PFA, 4\% (w/v) sucrose in phosphate-buffered saline for $15 \mathrm{~min}$ at room temperature (RT). Cells were permeabilized and blocked for $1 \mathrm{~h}$, RT in blocking buffer $(0.23 \%$ [v/v] Triton X-100, $385 \mathrm{mM} \mathrm{NaCl}$ in $15 \mathrm{mM}$ sodium phosphate buffer $\mathrm{pH} 7.4$, supplemented with $30 \%[\mathrm{v} / \mathrm{v}]$ goat serum or $10 \%[\mathrm{w} / \mathrm{v}]$ bovine serum albumin for staining with goat antibodies) before incubation with the specific primary antibody $(1 \mathrm{~h}, \mathrm{RT})$ and the secondary antibody (30 min, RT) in blocking buffer.

Immunocytochemistry of newly exocytosed proteins. Primary hippocampal neurons were transfected with c-myc epitope-tagged Syb2. On 14-15 DIV neurons were incubated with primary antibody in imaging buffer (20 min, RT). Neurons were washed with imaging buffer, stimulated in an electric field as described above and fixed with $4 \%(\mathrm{w} / \mathrm{v})$ PFA, $4 \%(\mathrm{w} / \mathrm{v})$ sucrose in phosphate-buffered saline for $15 \mathrm{~min}$ at RT. Further labelling steps were carried out in $15 \mathrm{mM}$ sodium phosphate buffer (pH 7.4), supplemented with $30 \%(\mathrm{v} / \mathrm{v})$ goat serum and $385 \mathrm{mM} \mathrm{NaCl}$. Primary antibodies on the neuronal surface were saturated with Alexa 488-labelled Fab fragments of secondary antibody (overnight, RT), followed by a labelling of newly exocytosed proteins with primary $(1 \mathrm{~h}, \mathrm{RT})$ and Alexa 647 labelled FAB fragments of secondary antibody ( $30 \mathrm{~min}, \mathrm{RT})$. Labelling of AP180 and Bassoon was performed with primary and secondary antibodies as described in the paragraph on immunocytochemistry.

Image acquisition of fixed hippocampal neurons. For acquisition of images from immunostainings without the need for improved resolution were used the inverted fluorescence microscope setup, described above. To reduce out of focus fluorescence in neuronal cultures that also contained astrocytes (Supplementary Fig. 3d) we used a laser-scanning microscope LSM780 from (Carl Zeiss Microscopy GmbH) equipped with an Argon-Laser 488 and an DPSS-Laser. Emission was detected at $500-550$ or $565-700 \mathrm{~nm}$.

Structured illumination microscopy. Hippocampal neurons were silenced for 5 min with $50 \mu \mathrm{M}$ APV and $10 \mu \mathrm{M}$ CNQX in imaging buffer, stimulated (40 APs $20 \mathrm{~Hz}$ ) or directly fixed and stained with primary and secondary antibodies as described before. Samples were mounted in Vectashield. 3D 3-colour SIM images were acquired using the 488, 568 and $643 \mathrm{~nm}$ laser lines, standard filter sets and $125 \mathrm{~nm} z$-sectioning of the OMX V4 Blaze (GE Healthcare) system. $100 \mathrm{~nm}$ fluorescent beads (Tetraspek, T7284, Invitrogen) were used for registration of detection channels achieving $<40 \mathrm{~nm}$ registration error for all three channels. Images were exported using Imaris 7.6 (Bitplane).

SD-dSTORM. Spectral-demixing (SD)-dSTORM provides registration error free, low crosstalk dual-colour super-resolution imaging with a resolution of $\sim 20-35 \mathrm{~nm}$ (refs 33,34). The system, acquisition and analysis procedure was described previously ${ }^{33}$. Here, we used Alexa Fluor 647 (Invitrogen) and CF680 (Biotium) as dye pairs distinguishable by spectral demixing ${ }^{34}$. The emission splitter was equipped with a dichroic mirror (700-DCXXR, AHF Analysentechnik) and an emission bandpass filter (F76-635, AHF Analysentechnik). The overlay of SD-dSTORM channels (NEP/AP180) with the wide-field channel (Bassoon) was done using a custom Image macro $^{52}$.

Generation of AP180/CALM-depleted neurons. AP180 expression was eliminated by gene knockout $(\mathrm{KO})$ in mice using homologous recombination: A floxed neomycin (NEO) resistance cassette was inserted in front of exon 3 and an additional loxP site after exon 6 in hybrid C57BL/6NTac x 129S6/SvEVTac ES cell clones injected into C57BL/6 blastocysts and implanted into pseudopregnant mice. Mating of chimeric males with Ella-Cre deleter mice removed exons 3-6 as well as the NEO cassette giving rise to heterozygous mice, which were interbred to obtain WT and AP180 ${ }^{-9}$ - littermates. Animals were genotyped by PCR analysis of genomic DNA (see Supplementary Fig. 3c). The AP180 WT allele was detected 
with the forward primer ACCTCATGTGAAACGTTGCCTG and the reverse primer TCTGGTGGATAGTGTCACTTAGGTAG (product: $323 \mathrm{nt}$ ). The AP180 $\mathrm{KO}$ allele was detected using the forward primer CCAGATGACCTGAGTTTGTG and the reverse primer TCTGGTGGATAGTGTCACTTAGGTAG (product: $401 \mathrm{nt})$.

Primary hippocampal neuron cultures prepared from AP180-I- mice (P0-P3) were transfected with pFUGW-based plasmids ${ }^{56}$ co-expressing mKate and specific shRNA directed against CALM ${ }^{28}$ to obtain AP180/CALM-depleted neurons. As a control, hippocampal neurons from WT mice (P0-P3) co-expressing mKate and scrambled control shRNA were used.

Plasmids and antibodies. Plasmids encoding for Syb2 (WT, rat) and Syt1 (rat) with a TEV protease cleavable superecliptic pHluorin-tag were a kind gift from J. Klingauf (University of Münster, Münster, Germany) ${ }^{7}$. Mutant Syb2 (M46A) was cloned into a pHluorin plasmid by PCR amplification and standard cloning procedures. A PCR based approach was used to introduce a c-myc epitope tag into a plasmid encoding Syb2 (WT). The Syp-pHluorin plasmid (rat) was a kind gift from L. Lagnado (University of Sussex, UK) ${ }^{48}$. A plasmid encoding Munc13-1mCherry (rat) was derived from pEGFP-N1 carrying Munc13-1, kindly provided by N. Brose (MPI for Experimental Medicine, Göttingen, Germany). Clathrin light chain (rat) was fused to mRFP and expressed from pcDNA5/FRT/TO and fused to eGFP, expressed from pcDNA3. The plasmid encoding for Lifeact-eGFP ${ }^{57}$ was a kind gift from F. Bradke (Center for Neurodegenerative Diseases, Germany). The CALM or scrambled shRNA was coexpressed with mKate from the pFUGW vector. The following antibodies were used: AP180 (155003, Synaptic Systems, 1: 100), Bassoon (141004, Synaptic Systems, 1: 500), CALM (sc-6433, Santa Cruz, 1: 100), clathrin heavy chain (ab21679, Abcam, 1: 400) c-myc epitope tag (9E10, purified from ascites, monoclonal, 1: 1,000).

Statistical analysis. If not indicated differently, traces were recorded from 4-12 neurons per individual experiment. From each neuron several tens to hundreds of synapses were analysed. Data was pooled in two steps: (i) by calculating the median from all synapses from one neuron; and (ii) by using this value to generate the median for all neurons within each individual experiment. $N$ individual experiments were averaged and used for statistical analysis. Maximal spread, $\mathrm{FWHM}^{2}$ of the last time point and the duration of half way reclustering were obtained from median filtered original traces in order to reduce noise. All values are depicted as mean \pm s.e.m. Statistical significance was calculated with Prism (Graphpad) using a two-way analysis of variance with repeated measures (for curves) or a two-tailed $t$-test (all bar graphs). Statistical significance is indicated as followed: ${ }^{* *}=P<0.01$; ${ }^{*}=P<0.05$.

\section{References}

1. Dittman, J. \& Ryan, T. A. Molecular circuitry of endocytosis at nerve terminals. Annu. Rev. Cell Dev. Biol. 25, 133-160 (2009).

2. Haucke, V., Neher, E. \& Sigrist, S. J. Protein scaffolds in the coupling of synaptic exocytosis and endocytosis. Nat. Rev. Neurosci. 12, 127-138 (2011)

3. Saheki, Y. \& De Camilli, P. Synaptic vesicle endocytosis. Cold Spring Harb. Perspect. Biol. 4, a005645 (2012).

4. Kononenko, N. L. \& Haucke, V. Molecular mechanisms of presynaptic membrane retrieval and synaptic vesicle reformation. Neuron 85, 484-496 (2015).

5. Royle, S. J. \& Lagnado, L. Clathrin-mediated endocytosis at the synaptic terminal: bridging the gap between physiology and molecules. Traffic 11, 1489-1497 (2010).

6. Ehlers, M. D., Heine, M., Groc, L., Lee, M. C. \& Choquet, D. Diffusional trapping of GluR1 AMPA receptors by input-specific synaptic activity. Neuron 54, 447-460 (2007).

7. Heine, M. et al. Surface mobility of postsynaptic AMPARs tunes synaptic transmission. Science 320, 201-205 (2008).

8. Kneussel, M., Triller, A. \& Choquet, D. SnapShot: receptor dynamics at plastic synapses. Cell 157, 1738-1738 e1731 (2014).

9. Levi, S. et al. Homeostatic regulation of synaptic GlyR numbers driven by lateral diffusion. Neuron 59, 261-273 (2008).

10. Fernández-Alfonso, T., Kwan, R. \& Ryan, T. A. Synaptic vesicles interchange their membrane proteins with a large surface reservoir during recycling. Neuron 51, 179-186 (2006)

11. Willig, K. I., Rizzoli, S. O., Westphal, V., Jahn, R. \& Hell, S. W. STED microscopy reveals that synaptotagmin remains clustered after synaptic vesicle exocytosis. Nature 440, 935-939 (2006).

12. Hua, Y. et al. A readily retrievable pool of synaptic vesicles. Nat. Neurosci. 14, 833-839 (2011).

13. Wienisch, M. \& Klingauf, J. Vesicular proteins exocytosed and subsequently retrieved by compensatory endocytosis are nonidentical. Nat. Neurosci. $\mathbf{9}$, 1019-1027 (2006).

14. Hosoi, N., Holt, M. \& Sakaba, T. Calcium dependence of exo- and endocytotic coupling at a glutamatergic synapse. Neuron 63, 216-229 (2009).
15. Neher, E. What is rate-limiting during sustained synaptic activity: vesicle supply or the availability of release sites. Front. Synaptic Neurosci. 2, 144 (2010).

16. Schmoranzer, J., Goulian, M., Axelrod, D. \& Simon, S. M. Imaging constitutive exocytosis with total internal reflection fluorescence microscopy. J. Cell Biol. 149, 23-32 (2000).

17. Sochacki, K. A. et al. Imaging the post-fusion release and capture of a vesicle membrane protein. Nat. Commun. 3, 1154 (2012).

18. Taraska, J. W., Perrais, D., Ohara-Imaizumi, M., Nagamatsu, S. \& Almers, W Secretory granules are recaptured largely intact after stimulated exocytosis in cultured endocrine cells. Proc. Natl Acad. Sci. USA 100, 2070-2075 (2003).

19. Miesenbock, G., De Angelis, D. A. \& Rothman, J. E. Visualizing secretion and synaptic transmission with $\mathrm{pH}$-sensitive green fluorescent proteins. Nature 394, 192-195 (1998).

20. Royle, S. J., Granseth, B., Odermatt, B., Derevier, A. \& Lagnado, L. Imaging phluorin-based probes at hippocampal synapses. Methods Mol. Biol. 457, 293-303 (2008).

21. Ferguson, S. M. et al. A selective activity-dependent requirement for dynamin 1 in synaptic vesicle endocytosis. Science 316, 570-574 (2007).

22. Darcy, K. J., Staras, K., Collinson, L. M. \& Goda, Y. Constitutive sharing of recycling synaptic vesicles between presynaptic boutons. Nat. Neurosci. 9, 315-321 (2006).

23. Wilhelm, B. G. et al. Composition of isolated synaptic boutons reveals the amounts of vesicle trafficking proteins. Science 344, 1023-1028 (2014).

24. Groc, L. et al. Surface trafficking of neurotransmitter receptor: comparison between single-molecule/quantum dot strategies. J. Neurosci. 27, 12433-12437 (2007).

25. Grote, E. \& Kelly, R. B. Endocytosis of VAMP is facilitated by a synaptic vesicle targeting signal. J. Cell Biol. 132, 537-547 (1996).

26. Koo, S. J. et al. SNARE motif-mediated sorting of synaptobrevin by the endocytic adaptors clathrin assembly lymphoid myeloid leukemia (CALM) and AP180 at synapses. Proc. Natl Acad. Sci. USA 108, 13540-13545 (2011).

27. Harel, A., Wu, F., Mattson, M. P., Morris, C. M. \& Yao, P. J. Evidence for CALM in directing VAMP2 trafficking. Traffic 9, 417-429 (2008).

28. Bushlin, I. et al. Clathrin assembly protein AP180 and CALM differentially control axogenesis and dendrite outgrowth in embryonic hippocampal neurons. J. Neurosci. 28, 10257-10271 (2008).

29. von Kleist, L. et al. Role of the clathrin terminal domain in regulating coated pit dynamics revealed by small molecule inhibition. Cell 146, 471-484 (2011).

30. Dutta, D., Williamson, C. D., Cole, N. B. \& Donaldson, J. G. Pitstop 2 is a potent inhibitor of clathrin-independent endocytosis. PLoS ONE 7, e45799 (2012).

31. Stahlschmidt, W., Robertson, M. J., Robinson, P. J., McCluskey, A. \& Haucke, V. Clathrin terminal domain-ligand interactions regulate sorting of mannose 6-phosphate receptors mediated by AP-1 and GGA adaptors. J. Biol. Chem. 289, 4906-4918 (2014).

32. Willox, A. K., Sahraoui, Y. M. \& Royle, S. J. Non-specificity of Pitstop 2 in clathrin-mediated endocytosis. Biol. Open 3, 326-331 (2014).

33. Lampe, A., Haucke, V., Sigrist, S. J., Heilemann, M. \& Schmoranzer, J. Multi-colour direct STORM with red emitting carbocyanines. Biol. Cell 104, 229-237 (2012)

34. Lehmann, M., Lichtner, G., Klenz, H. \& Schmoranzer, J. Novel organic dyes for multicolor localization-based super-resolution microscopy. J. Biophotonics. doi:10.1002/jbio.201500119 (2015).

35. Takamori, S. et al. Molecular anatomy of a trafficking organelle. Cell 127, 831-846 (2006).

36. van de Goor, J., Ramaswami, M. \& Kelly, R. Redistribution of synaptic vesicles and their proteins in temperature-sensitive shibire(ts1) mutant Drosophila. Proc. Natl Acad. Sci. USA 92, 5739-5743 (1995).

37. Roos, J. \& Kelly, R. B. The endocytic machinery in nerve terminals surrounds sites of exocytosis. Curr. Biol. 9, 1411-1414 (1999).

38. Teng, H., Cole, J. C., Roberts, R. L. \& Wilkinson, R. S. Endocytic active zones: hot spots for endocytosis in vertebrate neuromuscular terminals. J. Neurosci. 19, 4855-4866 (1999).

39. Denker, A. et al. A small pool of vesicles maintains synaptic activity in vivo. Proc. Natl Acad. Sci. USA 108, 17177-17182 (2011).

40. Saka, S. K. et al. Multi-protein assemblies underlie the mesoscale organization of the plasma membrane. Nat. Commun. 5, 4509 (2014).

41. Ceccarelli, B., Hurlbut, W. P. \& Mauro, A. Turnover of transmitter and synaptic vesicles at the frog neuromuscular junction. J. Cell Biol. 57, 499-524 (1973).

42. Zhang, Q., Li, Y. \& Tsien, R. W. The dynamic control of kiss-and-run and vesicular reuse probed with single nanoparticles. Science 323, 1448-1453 (2009).

43. Gordon, S. L., Leube, R. E. \& Cousin, M. A. Synaptophysin is required for synaptobrevin retrieval during synaptic vesicle endocytosis. J. Neurosci. 31, 14032-14036 (2011).

44. Kaempf, N. et al. Overlapping functions of stonin 2 and SV2 in sorting of the calcium sensor synaptotagmin 1 to synaptic vesicles. Proc. Natl Acad. Sci. USA 112, 7297-7302 (2015) 
45. Kwon, S. E. \& Chapman, E. R. Synaptophysin regulates the kinetics of synaptic vesicle endocytosis in central neurons. Neuron 70, 847-854 (2011).

46. Dason, J. S., Smith, A. J., Marin, L. \& Charlton, M. P. Cholesterol and F-actin are required for clustering of recycling synaptic vesicle proteins in the presynaptic plasma membrane. J. Physiol. 592, 621-633 (2014).

47. Ganguly, A. et al. A dynamic formin-dependent deep F-actin network in axons. J. Cell Biol. 210, 401-417 (2015).

48. Granseth, B., Odermatt, B., Royle, S. J. \& Lagnado, L. Clathrin-mediated endocytosis is the dominant mechanism of vesicle retrieval at hippocampal synapses. Neuron 51, 773-786 (2006).

49. Kononenko, N. L. et al. Clathrin/AP-2 mediate synaptic vesicle reformation from endosome-like vacuoles but are not essential for membrane retrieval at central synapses. Neuron 82, 981-988 (2014).

50. Watanabe, S. et al. Ultrafast endocytosis at mouse hippocampal synapses. Nature 504, 242-247 (2013).

51. Edelstein, A., Amodaj, N., Hoover, K., Vale, R. \& Stuurman, N. Computer control of microscopes using microManager. Curr. Protoc. Mol. Biol. Chapter 14, Unit14 20 (2010).

52. Schneider, C. A., Rasband, W. S. \& Eliceiri, K. W. NIH Image to ImageJ: 25 years of image analysis. Nat. Methods 9, 671-675 (2012).

53. Rowlingson, B. S. \& Diggle, P. J. Splancs-spatial point pattern-analysis code in S-Plus. Comput. Geosci. 19, 627-655 (1993).

54. Dixon, P. M. in Encyclopedia of Environmetrics. (Vol. 3. eds El-Shaarawi, A. H. \& Piegorsch, W. W.) 1796-1803 (John Wiley \& Sons, 2002).

55. Kiskowski, M. A., Hancock, J. F. \& Kenworthy, A. K. On the use of Ripley's $\mathrm{K}$-function and its derivatives to analyze domain size. Biophys. J. 97, 1095-1103 (2009).

56. Arancillo, M. et al. Titration of Syntaxin1 in mammalian synapses reveals multiple roles in vesicle docking, priming, and release probability. J. Neurosci. 33, 16698-16714 (2013).

57. Riedl, J. et al. Lifeact: a versatile marker to visualize F-actin. Nat. Methods 5, 605-607 (2008)

\section{Acknowledgements}

We thank Claudia Schmidt for technical assistance, and Drs Nils Brose (Max-Planck-Institute for Experimental Medicine, Göttingen, Germany), Jürgen Klingauf (University of Münster, Münster, Germany), and Leon Lagnado (University of Sussex, Brighton, UK) for the kind gift of plasmids. Supported by grants from the Deutsche Forschungsgemeinschaft (DFG HA2686/8-1 to V.H.; MA4735/1-1 to T.M.; SFB 958/Z02 to J.S.) and by a Max-Delbrück Center predoctoral fellowship (to N.G.).

\section{Author contributions}

N.G. and G.T. conducted all imaging experiments and data analysis; T.M. supplied knockout mice; J.S. and V.H. designed the study, analysed data; and together with N.G. wrote the manuscript.

\section{Additional information}

Supplementary Information accompanies this paper at http://www.nature.com/ naturecommunications

Competing financial interests: The authors declare no competing financial interests.

Reprints and permission information is available online at http://npg.nature.com/ reprintsandpermissions/

How to cite this article: Gimber, N. et al. Diffusional spread and confinement of newly exocytosed synaptic vesicle proteins. Nat. Commun. 6:8392 doi: 10.1038/ncomms9392 (2015).

(c) (i) This work is licensed under a Creative Commons Attribution 4.0 International License. The images or other third party material in this article are included in the article's Creative Commons license, unless indicated otherwise in the credit line; if the material is not included under the Creative Commons license, users will need to obtain permission from the license holder to reproduce the material. To view a copy of this license, visit http://creativecommons.org/licenses/by/4.0/ 